\title{
Konstruieren mit Teilebibliotheken unter dem System AutoCAD 13
}

\author{
Prof. Dipl.-Ing. Karin Jannasch
}

\section{Einleitung}

CAD-Systeme enthalten in der Regel nur allgemeine Elemente zur Bearbeitung von geometrischen Informationen. Die Nutzung wird aber erst rationell, wenn spezifische, oft wiederkehrende Formen und Objekte sowie Konstruktionsabläufe und Berechnungen benutzerfreundlich aufgerufen werden können. Deshalb erfährt die Frage nach anwenderspezifischen Erweiterungen von CAD-Systemen zunehmend Bedeutung.

Diese anwenderspezifischen Softwareanpassungen sind unerläßlich, will ein Unternehmen seine Entwicklungsarbeit erfogreich auf den Einsatz von CAD umstellen. Da zu diesem Zeitpunkt kaum Erfahrungen der Mitarbeiter mit CAD vorliegen können, sind vorerst die Systemanbieter gefragt. So stehen auf dem CAD-Markt eine Reihe von spezifischen Branchenlösungen zur Verfügung, die jedoch auf die ganz speziellen Probleme einzelner Unternehmen nicht ausreichend ausgerichtet sein können. Gute CAD-Systeme bieten darüber hinaus die Möglichkeit, eigene Softwareanpasungen vorzunehmen. Das erfordert aber die enge Zusammenarbeit zwischen dem späteren Nutzer des CAD-Systems und einem mit dem System vertrauten Programmierer. Da CAD-Händler dieser speziellen Aufgabe nicht gewachsen sind, ergab sich hier ein interessantes Betätigungsfeld für die TFH Wildau. Der folgende Bericht soll zeigen, welche Ergebnisse mit AutoCAD-Anpassungen im LG Konstruktion bisher erreicht wurden. Mein besonderer Dank gilt den Studenten des FB Maschinenbau Frau Britta Lange, Herrn Steffen Schlopsnies und Herrn Sven Lehmann, die bei der Bearbeitung der Industrieaufgaben wertvolle Unterstiitzung geleistet haben, sowie meinem Sohn André, der einen Teil der aufwendigen Systemprogrammierungen vorgenommen hat.

\section{Möglichkeiten der Systemerweiterungen}

Für die effiziente Arbeit mit einem CAD-System ist der Aufbau von leistungsfähigen Bibliotheken notwendig. Inhalte solcher Bibliotheken können sein:

- Zeichnungen im ACAD-Format *.DWG oder neutralem Format *.DXF:

Einzelteil- und Baugruppenzeichnungen

Kaufteile

Normteile

- Makros

wiederkehrende Formelemente

geometrische Grundformen
Angaben für Oberflächen, Schweißen, Härten ...

- Variantenprogramme

Parametrische Formelemente

Normteile in veränderlichen Abmessungen

Einzelteile mit verschiedenen parametrischen Formelementen

Einzelteile in variablen Größen

konstruktive Abläufe incl. Berechnungen

Die Bibliothekverwaltung, die Auswahl dieser Inhalte sowie das Einbinden in die Zeichnung muß durch ICONÜbersichten und geeignete Dialogfuihrung absolut benutzerfreundlich ablaufen.

\section{Beispiele}

\subsection{Menü zum Aufruf von Zeichnungsdateien}

Für die schnelle Auswahl der Ausstattung einer Yacht nach Kundenwünschen wurde für die Firma Maritime Technik GmbH Niederlehme das System AutoCAD13 erweitert /7/. Neben der Erstellung von Zeichnungen fuir spezielle Schiffsausruistungen ausgehend von alten Zeichnungsunterlagen und Prospektmaterial uiber Kaufteile mußte der Dialogführung spezielle Aufmerksamkeit geschenkt werden. Bild 1 zeigt eine Übersicht, wie die benutzerspezifische Steuerung von Dateiauswahl und Einfuigen in die Zeichnung erfolgen.

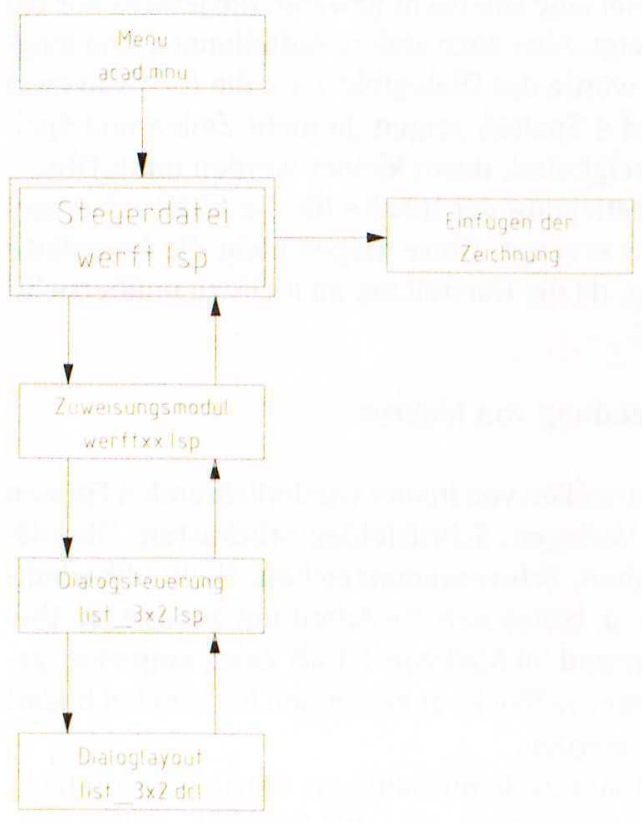

Bild 1: Auswahl und Einfügen von Dateien 
acad.mnu wird automatisch beim Start geladen werft.lsp AutoLISP-Steuerdatei, wird durch Eintrag in acad.lsp beim Start von ACAD geladen

lädt und startet den Zuweisungsmodul veranlaßt die Einfiigung der Zeichnung

werftxx.lsp Zuweisungsmodul lädt und startet den Dialog meldet den einzufügenden Dateinamen an die Steuerdatei

list_2×3.lsp Dialogsteuerungsdatei

meldet die Teileauswahl an den Zuweisungsmodul

list_3x2.dcl Dialoglayout

lädt und startet das Dialogfeld

Beim Anlegen von Teilebibliotheken ist folgendermaßen vorzugehen:

- Struktur der Teilegruppen festlegen

- einheitliche Layerbezeichnungen festlegen

- Einzelteilzeichnungen anfertigen

- Dia anlegen

$\begin{array}{ll}\text { TILEMODE } & \\ \text { MANSFEN } & 0,03,2 \\ \text { ZOOM } & G \\ \text { MBEREICH } & \\ \text { MACHDIA } & \end{array}$

- Erstellen der AUTOLISP-Steuerdatei

- Eintrag in der ACAD.LSP, damit die WERFT.LSP beim Start von ACAD geladen wird.

- Eintragung in ACAD.MNU

- Zuweisen der Zeichnungsnamen/Pfadangaben für die Dialogfelder

- Aufbau der Dialogfelder festlegen (Programmiersprache DCL)

- Laden des Dialogfeldes

Das Dialogfeld kann je nach Bedarf gestaltet werden. Um eine bessere Übersicht zu erreichen und möglichst viel Textinformationen anzeigen zu können, wurde in diesem Beispiel eine Übersicht gewählt, die jeweils nur ein ICON anzeigt. Aber auch andere Aufteilungen sind möglich, z. B. würde das Dialogfeld $2 \times 4$ die ICONs in zwei Zeilen und 4 Spalten zeigen. Je mehr Zeilen und Spalten angezeigt sind, desto kleiner werden die ICONs.

Für die Festlegung der Inhalte für die ICONs sind spezielle DIAs erzeugt. Diese zeigen nicht die komplette Zeichnung, da die Darstellung im ICON zu unibersichtlich wirkt.

\subsection{Verwendung von Makros}

Für das Darstellen von immer wiederkehrenden Formen wie z. B. Vorlagen, Schriftfelder, Stuicklisten, Oberflächenangaben, Schweißkennzeichen, Hydraulikschaltzeichen u. a. bietet sich die Arbeit mit Makros an. Diese Formen sind im Maßstab 1:1 als Zeichnung eingegeben, als externe Blöcke abgelegt und können bei Bedarf eingefuigt werden.

Die Handhabung kann dabei wesentlich vereinfacht werden, wenn statt des Befehls EINFüGE und Abfrage nach Dateispezifikation und Einfuigeoptionen die Auf- nahme eines zusätzlichen ACAD-Befehls vereinbart wird. Dieser Befehl (z. B. Rz für das Einfuigen eines Rauheitzeichens) löst eine Prozedur aus, die die entsprechende Datei findet, Faktoren für das Einfiigen festlegt, Textattribute (z. B. Rz 6,3) abfragt und das Makro einfügt. Solche zusätzlich vereinbarten Befehle werden iiber Tastatur eingegeben, können im Abrollmenui aufgenommen oder einem Meniifeld auf dem Tablett zugeordnet werden.

\subsection{Variantenkonstruktion für Getriebedeckel}

Die Gestaltung von Maschinenbauteilen orientiert sich an immer wiederkehrenden speziellen Formen, die sich aus dem Zusammenspiel der Einzelteile sowie deren Berechnung ergeben. Die Gestaltung eines Getriebelagerdeckels z. B. hängt von der allgemeinen Funktion sowie den Lagerabmessungen ab. Ausgehend von diesen Voraussetzungen werden die konkreten Deckel nach einem Algorithmus gestaltet, der immer wieder ähnliche Deckelformen erzeugt.

Das folgende Beispiel wurde für die Konstruktionsaufgabe Getriebeentwurf programmiert, um dem Studenten die Arbeit mit Variantenprogrammen zu demonstrieren.

Im konkreten Beispiel soll der Deckel folgende Funktionen erfuillen:

- Öl- und staubdichter Verschluß des Getriebekastens an der Lagerstelle

- Festlager-Deckel hat Kräfte in axialer Richtung zu uibertragen

- axiale Einstellung des Lagers

- Öl- und staubgedichte Öffnung für relativdrehendes Wellenende

Dabei sind folgende Abmessungen anderer Maschinenteile zu beachten:

- Abmessung der Lagerbohrung im Gehäuse

- erforderliche Anlagefläche (Schulterhöhe) für das Lager

- Platzbedarf für die Lagerinnenringbefestigung

- Größe und Anzahl der Befestigungsschrauben

- Abmessungen der Dichtung

Mit den in Bild 2 dargestellten vier Grundformen lassen sich Deckel für die betrachteten Funktionen gestalten. Je nach Lage der vorgegebenen Punkte 1 bis 2 wird der Deckel wie dargestellt oder gedreht/gespiegelt eingefuigt.

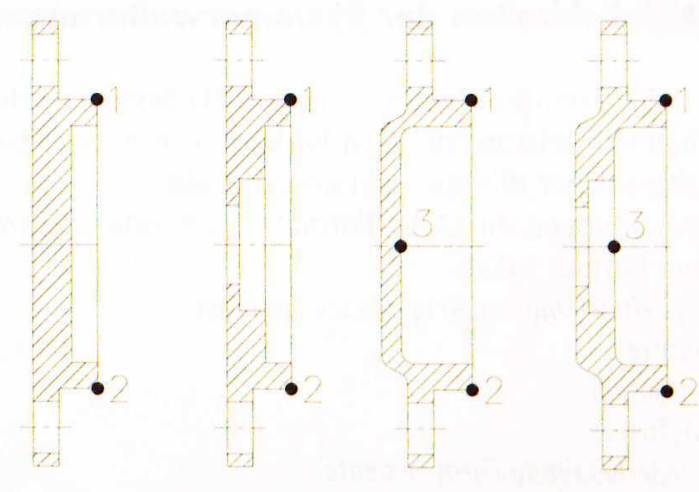

Bild 2: Deckelgrundformen 
Zum Aufruf der Deckelroutine unter ACAD wird ähnlich wie im Beispiel unter 3.1, Einfügen von Zeichnungen vorgegangen. Die Steuerdatei ist in diesem Fall mit DECKEL.LSP bezeichnet. Für dieses spezielle Beispiel wurde ein ICON-Rahmen mit einer Zeile und 4 Spalten verwendet.

Nach der Auswahl einer Deckelgrundform kann allerdings noch nicht auf eine einzufiigende Zeichnung wie im Beispiel Werft zurückgegriffen werden. Da unterschiedliche Parameter möglich sind, wird die Kontur über die Programmiersprache AutoLISP erzeugt.

Für die Erzeugung eines Deckels sind $\mathrm{z}$. B. folgende Angaben nötig:

\begin{tabular}{|c|c|}
\hline Parameter & Quelle \\
\hline $\begin{array}{l}\text { Durchmesser der Lager- } \\
\text { bohrung }\end{array}$ & Eingabe der Punkte 1 und 2 \\
\hline Lage normal & P 1 oben, $P 2$ unten \\
\hline gespiegelt & P 1 unten, P 2 oben \\
\hline horizontal & P1 links, P2 rechts \\
\hline Zentrierbundlänge & $\begin{array}{l}\text { intern als Funktion der } \\
\text { Lagerbohrung }\end{array}$ \\
\hline Schraubendurchmesser & Abfrage \\
\hline Lochkreisdurchmesser & $\begin{array}{l}\text { intern als Funktion von Lagerbohrung } \\
\text { und Schraubendurchmesser }\end{array}$ \\
\hline Außendurchmesser & $\begin{array}{l}\text { intern als Funktion von Lagerbohrung } \\
\text { und Schraubendurchmesser }\end{array}$ \\
\hline Ringfläche für Lagerschulter & Abfrage \\
\hline $\begin{array}{l}\text { Platzbedarf für Innenring- } \\
\text { befestigung } \\
\text { Bohrung für Dichtring, }\end{array}$ & Eingabe Punkt 3 \\
\hline Durchmesser & Abfrage \\
\hline Breite Dichtring & Abfrage \\
\hline Bohrung fuir Welle & Abfrage \\
\hline Breite des Deckels & $\begin{array}{l}\text { intern als Funktion vom Schrauben- } \\
\text { durchmesser }\end{array}$ \\
\hline Wölbung & intern als Funktion von der Lage des P 3 \\
\hline Fasen und Radien & intern als Funktion der Lạgerbohrung \\
\hline
\end{tabular}

Je nach Deckelgrundform ist ein Dialog zugeordnet, der die notwendigen Parameter abfragt. Als Funktion abgelegte Parameter werden intern berechnet. Sind alle Parameter vollständig, wird der Deckel in die Zeichnung eingefügt.

\subsection{Variantenprogramme aus Berechnungsdaten}

Für Bauteile, deren Parameter mit Berechnungsprogrammen bestimmt werden, kann eine spezielle Schnittstelle geschaffen werden, in der die für die Gestaltung erforderlichen Parameter abgelegt sind. Die Generierung der Grafik erfolgt wieder über ein AutoLISP-Programm, welches auf die Berechnungsdaten zurüickgreift. Im folgenden wird gezeigt, wie Getriebewellen und Zahnräder auf dieser Grundlage parametrisch erstellt werden können. Dieses Beispiel wurde ebenfalls für die Konstruktionsaufgabe Getriebeentwurf programmiert und ist Bestandteil des Programmes WCAD [6].

Für die Übergabe von Berechnungsdaten an AutoCAD und eine graphische Darstellung der berechneten Bauteile sind folgende Schritte erforderlich:

Die Welle wird in verschiedene Formelemente wie Viereck, Linie, Fase, Langloch und Text aufgeteilt und fortlaufend numeriert (s. Bild 3). Dabei kann sich ein Formelement, wie z. B. der Nabensitz von Rad 2 je nach konstruktiver Ausführung in der Lage und Form verändern.

Die Koordinatendaten für diese Formelemente werden nach ergänzenden Abfragen aus den Entwurfsdaten berechnet. Diese Elemente werden an die PASCAL-Unit interface iibergeben und in eine AutoLISP-Datei geschrieben.

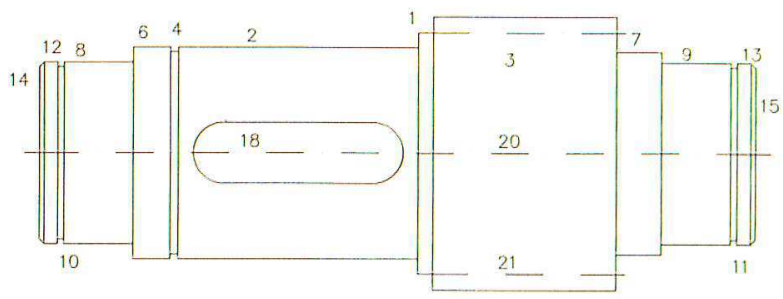

Bild 3: Definition der Formelemente

Die einzelnen Formelemente sind z. B. wie folgt zugeordnet:

$\begin{array}{lll}1 & \text { Bund } & \text { Viereck } \\ 2 & \text { Sitz des Zahnrades } & \text { Viereck } \\ 3 & \text { Sitz des Ritzels } & \text { Viereck } \\ 4,5,11,13 & \text { Einstich für Wellensicherungsring } & \text { Viereck } \\ 7,7,12,13 & \text { konstruktive Abstände } & \text { Viereck } \\ 14,15 & \text { Fase } & \text { Fase } \\ 18,19 & \text { Paßfedernut } & \text { Langloch } \\ 20 & \text { Mittellinie der Welle } & \text { Linie } \\ 21 & \text { Teilkreislinie } & \text { Linie } \\ \text { ab 30 } & \text { Texteintragungen } & \text { Text }\end{array}$

Für die verschiedenen möglichen Gestaltvarianten mußte von folgenden Fällen ausgegangen werden:

Antriebswelle und Abtriebswelle:

1. Kupplung rechts, Bund rechts, Ritzel rechts 4. Spiegelfall von 1. 2. Kupplung links, Bund rechts, Ritzel rechts 5. Spiegelfall von 2. 3. Kupplung links, Bund links, Ritzel rechts 6. Spiegelfall von 3.

Für den größten Wellendurchmesser am Bund wird ein Wert aus der Halbzeugtabelle für Rundmaterial ausgewählt. Dieser Durchmesser erscheint auch im Schriftfeld bei der Bezeichnung von Halbzeug/Werkstoff.

Räder werden in Halbschnitt/Halbansicht (s. Bild 4) dargestellt. Damit kann durch Löschen und Spiegeln bzw.

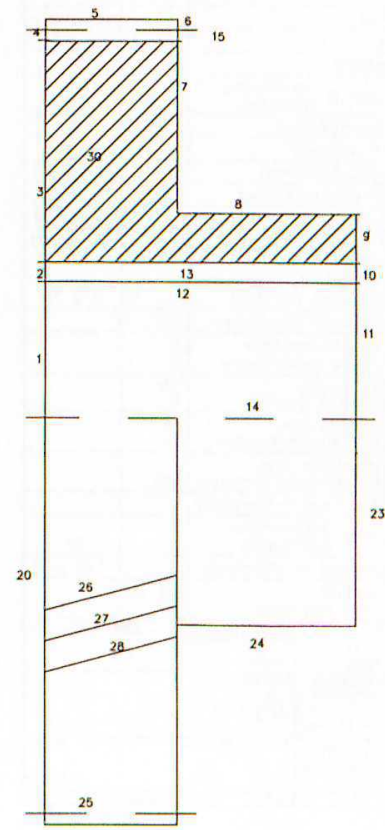

Bild 4: Definition der Formelemente am Zahnrad
Wahl der entsprechenden Layer das Rad in der Ansicht oder als Schnitt generiert werden. Auch hier wurden Formelemente vereinbart, die vor allem aus Linien und Text bestehen. Um die verschiedenen Radformen gestalten zu können und die Schraffur in ACAD zu gewährleisten, ist das Rad aus Teillinienstiicken zusammengesetzt.

Für diese Formelemente werden mit den entsprechenden Entwurfsdaten die erforderlichen Koordinaten berechnet. Für jedes dieser Formelemente existiert ein Unterprogramm, mit dem diese Daten in ein AutoLISP- 
Programm übersetzt und gespeichert werden. Je nach Gestaltungsvariante können dabei Spiegelungen erforderlich werden. Die gesamte Zeichnung wird danach auf dem Zeichenblatt zentriert.

Damit die Grafikdaten von AutoCAD aus aufgerufen werden können, mußte die AutoCAD-Datei ACAD.LSP geändert werden. Dadurch wird eine LISP-Prozedur erzeugt. Diese lädt das erforderliche Formblatt mit den Standardeinstellungen, stellt die notwendigen Dialogabfragen und öffnet die entsprechende LISP-Datei. In dieser befinden sich die eigentlichen Zeichenbefehle. Das Formblatt mit Zeichenblatt, Schriftfeld, Verzahnungstabelle, vorbereiteten Layern und Grundeinstellungen ist als DXF-Datei angelegt. Es stehen die Formate DIN A3 und DIN A2 mit oder ohne Verzahnungstabelle zur Verfügung. Die Zeichnungen werden grundsätzlich im Maßstab 1:1 angezeigt. Eintragungen im Schriftfeld und der Verzahnungstabelle werden so weit wie vorhanden eingetragen.

Die Bilder 5 und 6 zeigen Beispiele fuir die WCAD-Grafik einer Welle und eines Zahnrades.

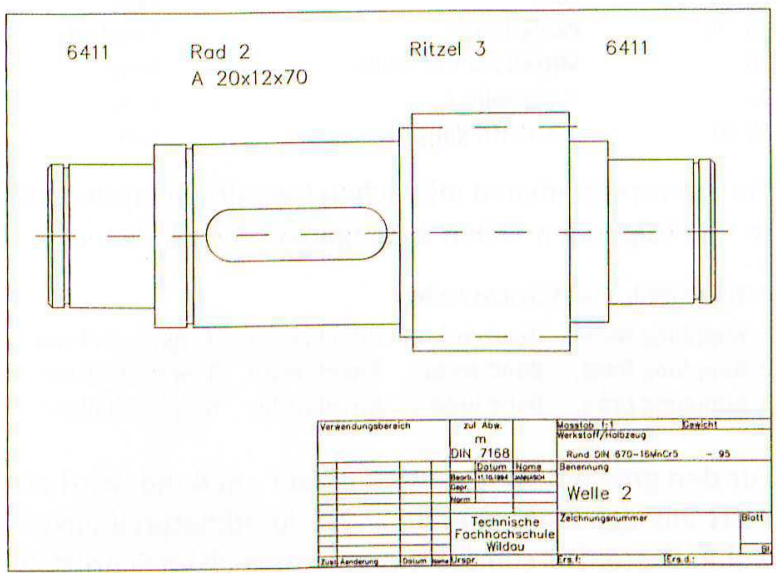

Bild 5: Zeichnung Welle

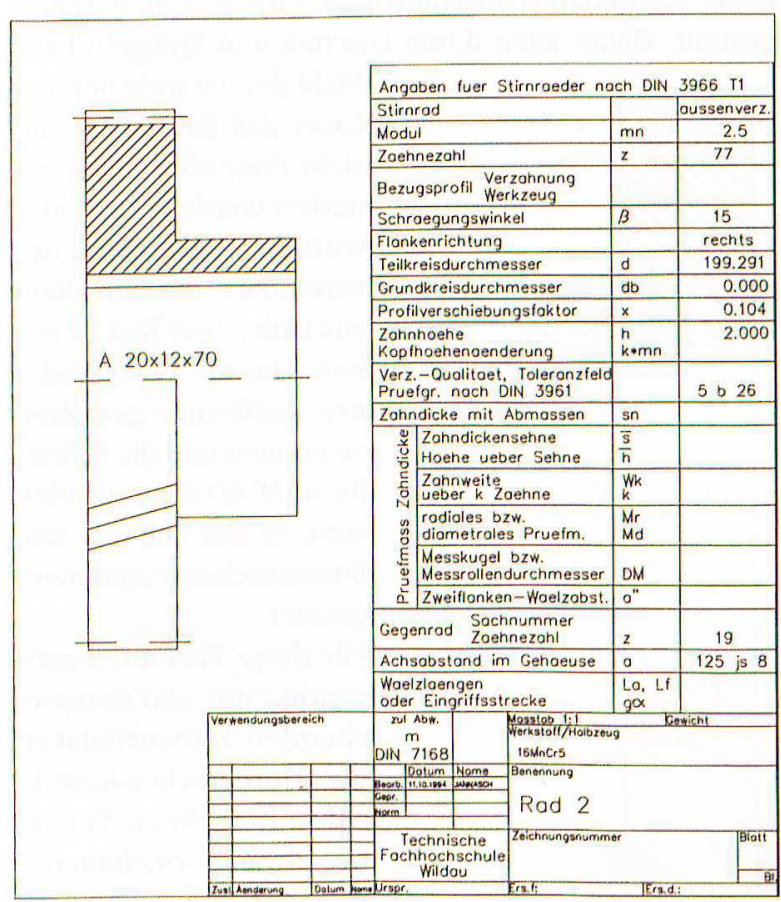

Bild 6: Zeichnung Zahnrad

\section{Zusammenfassung}

Unter dem System AutoCAD sind umfangreiche Systemerweiterungen möglich, die die benutzerfreundliche Handhabung erleichtern und die Effektivität der Konstruktionsarbeit erhöhen kann. Solche Erweiterungen sind systematisch zu planen und können nach einer gewissen Einarbeitung in die Systemprogrammierung von einem interessierten Mitarbeiter vorgenommen werden. Hinweise dazu bietet das Handbuch für Systemerweiterungen.

\section{Quellen}

[1] Vajna, CAD/CAM für Ingenieure, Vieweg Verlag 1994

[2] Eigner/Maier, Einstieg in CAD, Hanser Verlag München 1985

[3] Abeln, Die CA...-Techniken in der industriellen Praxis, Hanser Verlag München 1990

[4] ACAD Benutzerhandbuch, AutoDESK

[5] ACAD Softwareanpassungen, AutoDESK

[6] Jannasch, Computerunterstuitztes Entwerfen, Gestalten und Nachrechnen von Stirnradgetrieben in der Ingenieurausbildung, Wissenschaftliche Beiträge Heft 1, TFH Wildau 1995, S. 6-16

[7] Jannasch, Abschlußbericht über die Industrieaufgabe Maritime Technik GmbH Niederlehme - Einsatzvorbereitung für das CAD-System ACAD13, Januar 1996

\section{Verfasser}

Prof. Dipl.-Ing. Karin Jannasch

Technische Fachhochschule Wildau

Fachbereich Maschinenbau

Tel. (0 33 75) 507-161 